\title{
Glial fibrillary acidic protein (GFAP): modulation by growth factors and its implication in astrocyte differentiation
}

\section{F.C.A. Gomes ${ }^{1,2}$, \\ D. Paulin ${ }^{3}$ and \\ V. Moura $\mathrm{N}^{\text {eto }}{ }^{1}$}

\author{
${ }^{1}$ Departamento de Anatomia, Instituto de Ciências Biomédicas, and \\ 2Instituto de Biofísica Carlos Chagas Filho, \\ Universidade Federal do Rio de Janeiro, Rio de Janeiro, RJ, Brasil \\ ${ }^{3}$ Université Paris 7, Paris, France
}

\section{Correspondence \\ V. Moura Neto \\ Departamento de Anatomia ICB, UFRJ, CCS, Bloco F \\ 21949-590 Rio de Janeiro, RJ \\ Brasil \\ Fax: + 55-21-280-8193 \\ E-mail: vivaldo@ anato.ufrj.br \\ Presented at the 5th Brazilian Symposium on Extracellular Matrix - SIMEC, Angra dos Reis, \\ RJ, Brasil, September 7-10, 1998. \\ Research supported by PRO NEX-MCT (No. 052/97), CAPES-CO FECUB, FAPERJ, FINEP, CEPG-UFRJ.}

Received December 7, 1998 Accepted January 13, 1999

\begin{abstract}
Intermediate filament (IF) proteins constitute an extremely large multigene family of developmentally and tissue-regulated cytoskeleton proteins abundant in most vertebrate cell types. Astrocyte precursors of the CNS usually express vimentin as the major IF. Astrocyte maturation is followed by a switch between vimentin and glial fibrillary acidic protein (GFAP) expression, with the latter being recognized as an astrocyte maturation marker. Levels of GFAP are regulated under developmental and pathological conditions. Upregulation of GFAP expression is one of the main characteristics of the astrocytic reaction commonly observed after CNS lesion. In this way, studies on GFAP regulation have been shown to be useful to understand not only brain physiology but also neurological disease. Modulators of GFAP expression include several hormones such as thyroid hormone, glucocorticoids and several growth factors such as FGF, CNTF and TGFß, among others. Studies of the GFAP gene have already identified several putative growth factor binding domains in its promoter region. Data obtained from transgenic and knockout mice have provided new insights into IF protein functions. This review highlights the most recent studies on the regulation of IF function by growth factors and hormones.
\end{abstract}

\section{Introduction}

The cytoplasm of animal cells is structured by a scaffolding composed of microfilaments (MF), microtubules (MT) and intermediate filaments (IF). MF and MT are constituted by actin and tubulin, respectively, in all types of cells. Unlike MT and MF, IF proteins are regulated developmentally and tissue-specifically. The functional basis of this diversity is not well understood; however, changes in IF expression occur sequen- tially, coincidentally with changes in cellular differentiation states. The differential expression of IF proteins in different cell types implies that they have specialized roles in the differentiation and function of these cells. At present, proteins that make up the IF family can be classified into five distinct types (Table 1) (for a review, see Ref. 1). Class I and class II IFs consist of acidic and neutral-basic cytokeratins, respectively. These proteins are present in most epithelial cells and are, perhaps, the most divergent and atypical of all 
IF proteins. Class III IFs are formed by proteins expressed in different tissues: glial fibrillary acidic protein (GFAP), characteristic of astroglial cells; desmin, present in muscle cells; synemin, in certain muscles; peripherin, specific for the peripheral nervous system as well as for some central nervous system (CNS) neurons, and vimentin, present in all mesenchymal tissues. The pattern of vimentin expression differs from that of other IFs, since the protein appears transiently in a wide variety of cells during development.

Classe IV IF proteins are formed by neurofilaments (NF) and internexin present in neurons and by nestin, present in precursors of the nervous system (NS) and muscles. The lamins, lining the inner surface of the nuclear membrane, represent the class V IF ubiquitously expressed throughout most cell types.

Recently, new classes of IFs have been described: filensin and phakinin, named the beaded filaments, a novel class of IFs pres-

Table 1 - Types and cellular distribution of intermediate filamentsa.

alntermediate filament proteins have been classified into 5 main types according to structure and primary sequence similarities. bSome of them, recently described, have not yet been classified (nc), and are called the beaded filaments. CIF proteins are normally cell-specific, except for vimentin which can be expressed in several cell types usually before another IF protein is expressed. GFAP, Glial fibrillary acidic protein.

\begin{tabular}{lll}
\hline Intermediate filament & Class & Cell type \\
\hline Acid cytokeratins & I & Epithelial cells \\
Neutral-basic cytokeratins & II & Epithelial cells \\
Vimentinc & III & Mesenchymal cells \\
GFAP & III & Astroglial cells \\
Desmin & III & Muscle cells \\
Synemin & III & Skeletal muscle cells \\
Peripherin & III & Neurons \\
Nestin & IV & CNS and muscle precursor cells \\
Neurofilaments & IV & Neurons \\
Internexin & IV & Neurons \\
Filensin, phakininb & nc & Lens \\
Transitin & nc & Radial glial cells \\
Lamins & V & Ubiquitous
\end{tabular}

ent in the lens (2) and, more recently, transitin, a novel IF protein that is transiently expressed by radial glia during CNS development. This protein is expressed by midline radial glia structures, by several axon commissures and by Bergmann glia of the developing cerebellum (3).

Despite their great diversification throughout tissues, IF proteins present an evolutionary conservation of their structures. Three distinct domains can be identified in the structure of the IF proteins: a central rod of conserved length and sequence occurring in a helical conformation, flanked by a nonhelical N-terminal headpiece of variable length and a non-helical C-terminal tail showing considerable length differences between different IF proteins.

The organization of IF proteins into a complex network, their interaction with MTs and MFs to form the cytoskeleton and their appearance according to a highly specific developmental profile, all indicate an important biological role of IF filaments. However, since their first description, a great deal of discussion on the possible function of IFs exists in the literature. The availability of molecular genetic tools, such as dominant negative or knockout mutants in IF genes and transgenic mice, has provided a new avenue to explore the significance of IF proteins (for a review, see 4).

Except for keratins and desmin, for which knockout studies have clearly demonstrated a biological role $(4,5)$, there are conflicting data about the function of the remaining IF proteins. Whereas targeted disruption of the NF gene impaired the regeneration of myelinated axons following crush injury of peripheral nerves (6), introduction of a null mutation of the vimentin gene into the germ line of mice has produced healthy animals without an obvious phenotype (7). Similar results were obtained by disruption of the GFAP gene which did not cause defects in CNS development (8). However, as we will comment later, a recent null mutation of the 
GFAP gene disrupted the integrity of CNS white matter and caused abnormal myelination (9) as well as inefficient blood-brain barrier formation (10).

All of these data contribute to the controversy surrounding the functions of IF proteins. In this regard, the CNS has been extensively studied concerning IF functions. In this review, we will focus on CNS IFs by discussing the most recent advances in IF gene regulation and its implication in astrocyte and CNS development.

\section{Central nervous system and astrocytes}

The vertebrate nervous system is formed mainly by two heterogeneous classes of cells which are neurons and glia. Since the description and acceptance of the Neuron Doctrine nearly a century ago, neurons were regarded as the unique functional element of the nervous system. Glial cells, of which astrocytes constitute an important part, were described by Virchow in the middle of the nineteenth century as a merely supportive structural element of the nervous system. The astrocytes comprise a heterogeneous family of morphologically and functionally distinct cells whose structural plasticity is maintained mostly by a filamentous network consisting mainly of vimentin and GFAP.

Since the description of astrocytes, the idea of a passive structural element of Virchow has been gradually replaced by a more dynamic picture in which the glial network may actively take part in neuronal physiology.

Evidence accumulated in the past years demonstrates that astrocytes have a variety of active roles in maintaining normal brain physiology such as secretion of several active compounds, formation of the blood brain barrier, metabolism of several neurotransmitters and maintenance of the ionic balance of the extracellular medium (11).

Radial glia, an astrocyte precursor, is involved in neuronal migration during embryonic brain development (12). Glial cells, in general, are also involved in providing neurotrophic signals to neurons required for their survival, proliferation and differentiation $(13,14)$.

Besides their physiological role, astrocytes play an important role in pathological conditions of the nervous system. Accumulation of glial fibers is the histological landmark of the astrocyte response to NS injury, appropriately named reactive gliosis. Such response is characterized by intense astrocyte proliferation and overexpression of GFAP, among others. The fact that glial scars are frequently found in several NS disorders, such as multiple sclerosis, heavily implicates the astrocytic cells not only in the physiology of the NS as well as in neural disease development (15)

\section{Intermediate filaments in astrocytes: GFAP and vimentin}

In the vertebrate nervous system, neuroepithelial multipotent stem cells which are morphologically homogenous at the beginning of neurulation subsequently give rise to a variety of cell types, including neurons and astrocytes (16). One of the main hallmarks of developmental neurobiology is to understand the molecular mechanisms by which such cellular diversity is generated. Such diversification occurs at an early stage of development, especially by activation of sets of cell type-specific genes, which gives cells distinctive functions and morphological characteristics. Some of these cell-specific genes are the IF protein genes, which are regulated during cell development.

The two major IF proteins of astrocytes are vimentin and GFAP. In the course of astrocyte development, a transition in the expression of IF protein genes is observed. Early during development, radial glia and immature astrocytes express mainly vimentin (15). Towards the end of gestation, a switch 
occurs whereby vimentin is progressively replaced by GFAP in differentiated astroglial cells $(17,18)$.

At present, there is no consensus on the functional role of these IF proteins. The application of molecular genetic approaches to IF function has been providing some significant insights as well as raising new questions about the functional role of individual IF proteins.

\section{Vimentin}

Vimentin IFs are the only IF type found in a variety of cells including astrocytes, fibroblasts, endothelial cells, macrophages, neutrophils and lymphocytes (19). Functional analysis of the vimentin gene promoter has already been carried out and several negative and positive elements were identified within this region (20).

Data obtained from vimentin knockout mice (-/-) demonstrated that those animals developed and reproduced without presenting an obvious new phenotype, thus heavily calling into question the biological function of vimentin (7). Several data, however, argue in favor of a relevant function for vimentin. Using the same vimentin (-/-) lineage as Colucci-Guyon et al. (7), Galou et al. (21) found that GFAP filaments were also absent in certain glial cells that normally coexpress vimentin and GFAP such as the Bergmann glia and an astrocyte subpopulation of the corpus callosum. This was not due to the inability to express GFAP. Transfection of cultured vimentin -/- astrocytes with a vimentin cDNA restores the vimentinGFAP filament network, suggesting that in these cells vimentin might be required for coassembly with GFAP filaments (21).

\section{Glial fibrillary acidic protein}

Initially isolated from multiple sclerosis plaques, GFAP has been widely recognized as an astrocyte differentiation marker, con- stituting the major IF protein of mature astrocyte (22).

The developmental schedule of GFAP expression is not known in detail. In the mouse CNS, GFAP expression has been first detected at the end of gestation (18). Transcriptional studies demonstrated that GFAP mRNA increases between birth and day 15 and then decreases until day 55 (23). After reaching a plateau lasting into the second year of adult life, GFAP mRNA and protein levels tend to increase again in some regions such as the hippocampus, striatum and cortex (24). This usually corresponds to the increase of reactive astrocytes. Such increase during senescence is one of the most generalized markers for brain aging.

Insights into the role of GFAP have only recently emerged with reports on subtle abnormalities in GFAP-deficient-mice $(8,9)$. The first report on GFAP-/- mice emphasized that these mice lacked gross neurologic or behavioral abnormalities, with astrocytes lacking GFAP presenting a normal distribution (8). Although these studies call into question the role of GFAP in brain development, several subsequent papers heavily contradicted these previous results. Liedtke et al. (9) reported abnormal myelination in the optic nerve and reduced myelin thickness in the spinal cord of mutant mice carrying a null mutation in the GFAP gene. White matter was poorly vascularized and the blood brain barrier was structurally and functionally impaired. This work led to a new concept about GFAP function which is a novel link between astrocyte function and myelination. Recently, Pekny et al. (10) have shown that primary cultures of GFAP-/- astrocytes exhibited an increased final cell saturation density. Those results led the authors to speculate that the loss of GFAP expression observed focally in a proportion of human malignant gliomas might reflect tumor progression to a more rapidly growing and malignant phenotype. Consistent with this finding is the fact that some astrocyte-de- 
rived brain tumors lack GFAP immunoreactivity. In fact, activation of the GFAP gene promoter has recently been related to decreased astrocyte proliferation (25).

Some data indicate a novel concept that GFAP might play an important role in the control of neurological disease. Liedtke et al. (26) analyzed the astroglial response in GFAP-/- mice with experimental autoimmune encephalomyelitis, a model for multiple sclerosis. GFAP-/- astrocytes presented a disorganized cytoarchitecture due to irregular spacing and a decreased number of hemidesmosomes. The disease was more severe in mutant mice than in control animals, with conspicuously ill-defined and infiltrative lesions in GFAP-/- mice, thus adding weight to the concept that structural stabilization of white matter through filamentcompetent astrocytes is a crucial factor in limiting the lesion.

Although there is still some controversy about GFAP function in brain physiology and pathology, a large amount of evidence has been accumulating in the past few years in favor of an active and relevant role for this structural intermediate filament protein in brain development.

\section{Growth factor and hormone regulation of astrocyte differentiation}

GFAP synthesis is considered an important element of the developmental program of astrocyte differentiation and is part of the reactive response to almost any CNS injury. Thus by determining how transcription of the GFAP gene is controlled in these circumstances, insights should be obtained into the more general mechanisms of CNS development and disease. Indeed several putative growth factor binding domains have already been identified in the GFAP gene promoter region (Figure 1). This section will summarize the most relevant hormones and growth factors identified until now involved in modulating GFAP expression and astrocyte matu- ration in physiological and pathological conditions.

\section{Thyroid hormone}

Thyroid hormones are known to be important for normal brain development. Experiments in vivo have shown that neonatal treatment of rats by subcutaneous injections of T3 affects the development of astroglia in forebrain and hippocampus, accelerating the transition from vimentin-positive to GFAPpositive cells in both brain regions (27). In addition, the density of GFAP-positive cells, as well as the intensity of immunoreactivity, was substantially increased (27).

In fact, we have reported that $\mathrm{T} 3$ treatment induces cortical astrocytes which presented a flat morphology in vitro to become process-bearing cells $(28,29)$. This morphological change was followed by an increase in the GFAP content in midbrain and cerebral hemisphere astrocytes $(28,30)$ and an increased secretion of 8 -kDa peptides like insulin growth factor. In addition to an increased GFAP content, there was also a reorganization of the GFAP network. In control

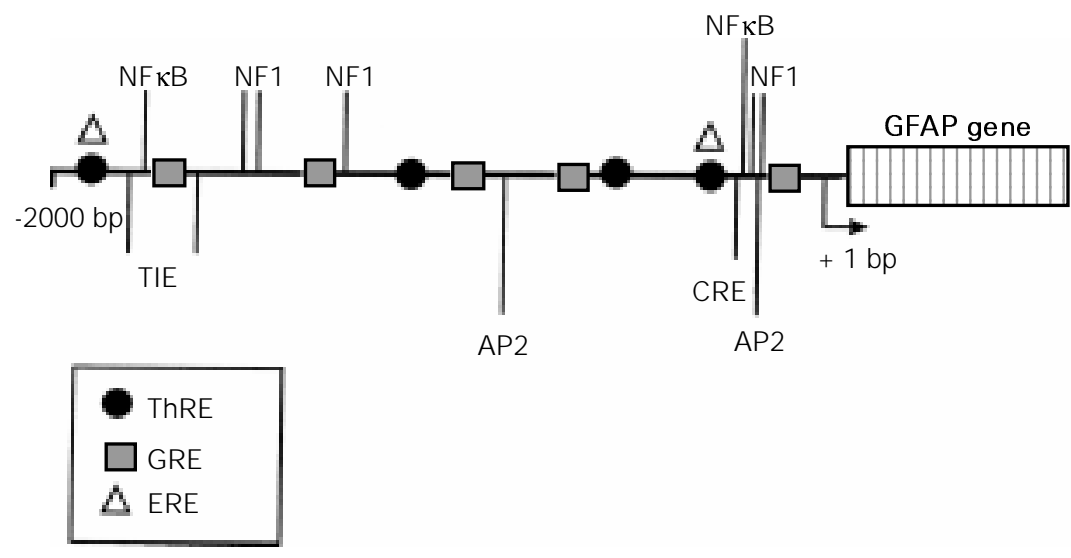

Figure 1 - Putative response elements in GFAP gene promoter. The hormone putative response elements in the rat glial fibrillary acidic protein (GFAP) gene promoter are indicated by symbols: black circles, ThRE, thyroid hormone response factor element; white triangles, ERE, estrogen response element; gray squares, GRE, glucocorticoid response element. Other putative transcription factor binding sites are indicated by the vertical lines: NF1, nuclear factor 1; AP2, activator protein 2; TIE, TGF-ß inhibitory element; CRE, CAMP response element; NFאB, nuclear factor $\kappa B$. The GFAP gene promoter and its consensus binding sites were not depicted on scale. Some putative domains were omitted (for details, see reference 24). bp, Base pair. 
cultures, GFAP gliofilaments displayed a diffuse intermediate filament network starting from the perinuclear region, present throughout the cytoplasm and reaching the cell membrane. Treated astrocytes clearly showed a reorganization of the gliofilaments which seemed to be more condensed in the astrocytic process (Figure 2).

Unlike cortical astrocytes, cerebellar astrocytes did not alter their morphology in response to T3. Rather, those astrocytes proliferate due to hormonal treatment. T3 did not increase GFAP synthesis in cerebellar astrocytes in vitro although it promoted alteration in GFAP organization. Treated cultures presented a preferential perinuclear organization of GFAP filaments with a small cytoplasmic array (31).

Putative thyroid hormone response element has already been identified in the GFAP gene promoter (Ref. 24 and Figure 1). However, the activity of this element remains to be proved. Indeed, we have demonstrated an alternative pathway of $\mathrm{T} 3$ hormone action

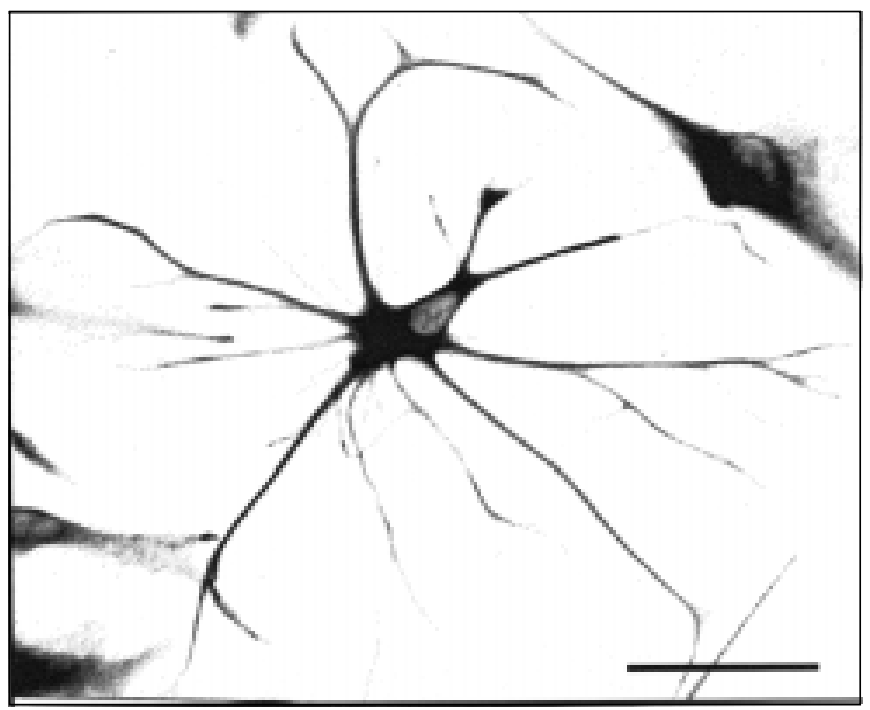

Figure 2 - Morphological changes induced by thyroid hormone in astrocyte cultures. Cerebral hemisphere astrocytes were grown in the presence of fetal calf serum until confluence. Cultures were then maintained for 3 days in the presence of $50 \mathrm{nM}$ T3 in serum-free medium, which was changed daily. Four days after T3 treatment, primary cultures were subcultured for $24 \mathrm{~h}$ and astroglial morphology was evaluated by immunocytochemistry for GFAP (29). T3-treated astrocytes presented a stellate morphology with GFAP-enriched processes. Scale bar, $50 \mu \mathrm{m}$. on astrocytic cells. T3 can induce GFAP synthesis and astrocyte differentiation by promoting synthesis and secretion of growth factors which themselves can act autocrinally on astrocytic cells (28).

Furthermore, T3 treatment induces cerebellar astrocytes to secrete growth factors which, besides acting autocrinally on astrocytic cells as commented above, can paracrinally act on neuronal cells. In fact, we have recently identified some of these secreted factors as epidermal growth factor (EGF) and tumor necrosis factor $\beta$ (TNFß), which induce neuronal proliferation (13). This is a novel pathway for the action of thyroid hormone on neurons via astrocytic cells.

\section{Steroid hormones}

There is evidence that glucocorticoids inhibit GFAP expression both at the mRNA or protein level in vivo $(32,33)$. However, there are contradictory results regarding the effects of glucocorticoids on GFAP expression in vitro. Astrocyte cultures from neonatal rats generally show an increase in GFAP expression when treated with corticosteroid (34). Glucocorticoid response element has been identified in the GFAP gene promoter with a putative functional significance (Figure 1 and Ref. 24).

Previous studies have demonstrated that estradiol promotes differentiation of glial cells from a flat morphology to a process-bearing one. Such morphological change is followed by a redistribution of GFAP in hypothalamic astrocytes in vitro and is dependent on the presence of neuronal cells (35).

Gonadal steroids have also been shown to influence GFAP immunoreactivity in the rat brain. As we have already discussed, GFAP levels are highly increased in various regions of senescent rat brains (24). Increased GFAP was associated with lower testosterone concentration in older rats (24). According to these results, Day et al. (36) recently reversed 
the age-related increase in GFAP by supplying rats with exogenous testosterone, thus supporting a role for sex steroids in GFAP regulation and astrocyte differentiation.

\section{Fibroblast growth factors}

Initially described by Trowell and collaborators in the 1930's as a mitogenic activity of pituitary and brain extracts on fibroblasts, fibroblast growth factors (FGF) are now widely recognized for their effects on a large spectrum of cells. The FGF family consists of 2 components called acidic FGF (aFGF) and basic FGF (bFGF), which share $60 \%$ homology.

The FGFs have been shown to play an important role in the survival and proliferation of neuroectodermal cells, as well as CNS glia and neurons $(37,38)$. Recently, Qian et al. (37) demonstrated that bFGF concentration regulates the generation of neurons and glia from multipotent cortical stem cells. In vivo neurogenesis is dramatically inhibited by neutralizing antibodies to bFGF (39).

The levels of bFGF are markedly increased in vivo after brain injury and in tissue specimens from several brain tumors, suggesting that this growth factor may play a role in nerve regeneration and tumor formation $(40,41)$. However, the role of FGF in tumorigenesis is still debated. Recently, Holland and Varmus (42), using a glia-specific gene transfer in mice, demonstrated that bFGF induces proliferation and migration of glial cells in vivo without the induction of tumors.

\section{Inflammatory cytokines}

The variety in the glial response suggests that multiple signals might be involved, eliciting different effects. Among the candidates for such effects are the members of the cytokine family of intercellular mediators whose levels usually increase with damage or disease to the nervous system. Such family includes interleukin 1 (IL-1), interleukin 6 (IL-6), leukemia inhibitory factor (LIF), tumor necrosis factor alpha (TNF $\alpha$ ), interferon gamma (IFN $\gamma$ ), transforming growth factor $\beta$ (TGFß), and most recently ciliary neurotrophic factor (CNTF).

\section{Ciliary neurotrophic factor}

Originally isolated as a survival neurotrophic factor for chick ciliary ganglion neurons, CNTF is now known to have pleiotropic actions on many neuronal and glial cells. This factor has high sequence homology with IL-6 family members, including IL-6, leukemia inhibitor factor (LIF) and oncostatin M (43).

A great deal of both in vivo and in vitro evidence has accumulated supporting a role of CNTF in the astrocyte response to injury. Intracerebral injections of CNTF into glial cells in vivo evoke a strong astrogliotic response with increases in GFAP content (44). GFAP-positive cells colocalize with immature astrocytes and not with oligodendrocyte cell specific markers, suggesting that CNTF upregulates GFAP mRNA in a preexisting immature astrocyte cell population. Seven putative and novel CNTF responsive elements have been identified within the proximal and distal elements of the GFAP gene promoter $(43,44)$. Furthermore, Clatterbuck et al. (45) reported that in vivo CNTF increases GFAP expression and induces concomitant morphological changes in CNS astrocytes by a direct action of CNTF on the GFAP gene promoter.

The fact that CNTF mRNA is increased after a lesion of the CNS supports a role for this factor in the response to injury. Intracerebral injection of recombinant human CNTF into the neocortex of adult rat induced a volume increase of the somas and the nuclei of protoplasmic and fibrous astrocytes in vivo (46), a hypertrophy characteristic of reactive gliosis indicating a putative 
role of CNTF in the astrocyte response to CNS lesion.

\section{IL-1, IL-2 and IL-6}

The function of IL-1 in astrocyte differentiation and GFAP expression is still unclear. IL-1 injection into adult or neonatal mouse brain results in reactive astrocytes with robust GFAP-bearing processes (47). However, in cultured astrocytes, IL-1 decreases GFAP mRNA and has no effect on GFAP protein (48). Injection of IL-2 and IL6 have been reported to induce GFAP protein in neonatal mice (47). Acute astrocytic responses to IL- 6 in mice and rats are consistent with the extensive astrocytosis in transgenic mice that overexpress IL-6 in astrocytes (49). Those data are in agreement with recent findings that absence of IL- 6 in genetically IL-6-deficient mice led to a massive reduction in the number of activated GFAP-positive astrocytes (50).

\section{Leukemia inhibitory factor}

Several data strongly implicate the LIF family of cytokines in the regulation of astrocyte differentiation, especially following NS injury $(51,52)$. Recently, Koblar et al. (51) reported that neural precursors isolated from the developing forebrain of low affinity leukemia inhibitory factor receptor (LIFR-/-)-deficient mice fail to generate astrocytes expressing GFAP when cultured in vitro. These data support in vivo findings that show extremely low levels of GFAP mRNA in brains of embryonic LIFR-/- mice. Taken together, these findings indicate that LIF signaling might be required for the expression of both functional and phenotypic markers of astrocyte differentiation.

\section{Tumor necrosis factor $\alpha$}

$\mathrm{TNF} \alpha$ is a pluripotent cytokine report- edly mitogenic to astrocytes. TNF $\alpha$ treatment resulted in a decrease in GFAP content and its encoding mRNA in mature bovine secondary astrocytes (53). A reduction of GFAP mRNA by TNF was also reported by Oh et al. (48) in TNF-treated secondary mouse astrocyte cultures, although these authors did not find alterations in the GFAP protein content.

Murphy et al. (54) have also reported a decrease in GFAP content and its encoding mRNA in U373 human glioblastoma cells and primary mouse astrocyte cultures treated with TNF $\alpha$. The extent to which changes in GFAP expression are linked to cell division is not clear. However, TNF $\alpha$ treatment resulted in a larger decrease in GFAP content when the cells were subconfluent, which might suggest a negative correlation between GFAP expression and TNF-induced proliferation. Although this hypothesis is still unclear, results obtained with GFAP-transgenic mice recently demonstrated that astrocyte induction of the GFAP gene promoter and astrocyte differentiation by neuronal cells were followed by a decrease in astrocyte proliferation, suggesting that quiescence in response to neurons might be a relevant feature for the astrocyte differentiation pathway (25).

\section{Transforming growth factor $B$}

The TGFß superfamily is composed of a range of functional and structural factor subclasses with predominantly growth-inhibitory cellular actions and developmental regulatory effects on organogenesis, pattern formation, modulation of extracellular matrix and terminal differentiation (55). The subclasses include the TGFß, activins, glialderived factors (GDFs), Mullerian inhibiting substances, glial-derived neurotrophic factor (GDNF), cartilage-derived morphogenetic proteins (CDMPs) and the rapidly expanding factor subclass of bone morphogenic proteins (BMPs). A range of TGF $\beta$ family members is expressed in the NS and 
exhibit a spectrum of regulatory actions on neural cells during development. TGFß isoforms exhibit a complex range of cellular actions on the astroglial lineage which depends on the state of astrocyte differentiation (55). TGFß is reported to inhibit microglial and astrocyte proliferation (56) and to induce the expression of GFAP in vivo (57) and in vitro (24). The fact that TGFß mRNA and protein are highly enriched in glial scars after cortical stab wounds suggests a role for such factor in GFAP regulation and scar formation during reactive gliosis (for a review, see 24).

\section{Bone morphogenetic proteins}

BMPs participate in a broad spectrum of cellular inducing events involving all three germ layers during metazoan development. This subclass of the TGF $\beta$ superfamily has only recently gained attention in the study of mammalian CNS development. BMPs are expressed in multiple CNS regions throughout development and may regulate additional early neural cellular processes and participate in many aspects of neuronal maturation (58). Gross et al. (59) have reported that various BMPs induce multipotent neuronal progenitor cells derived from the embryonic subventricular zone to differentiate into astrocytes. Such BMP-mediated astroglial inductive action is associated with a nearly complete suppression of neuronal and oligodendroglial genes and was followed by an enhanced morphological complexity and expression of GFAP. Similarly, Mabbie et al. (60) have recently reported that BMPs promote the selective differentiation of $0-2 \mathrm{~A}$ progenitor cells to astrocytes with a concurrent increase in GFAP-immunoreactive cells and suppression of oligodendroglial genes. These results suggest that BMPs might have a selective role in determining astrocytic lineage commitment, possibly regulating astrocyte specific genes such as the GFAP gene.

\section{Neuron-glia interactions: implications for GFAP expression}

During CNS development, neurons are born on the ventricular surface of the neural tube and appear to migrate to their final destination (12). Thus it is assumed that structural support of astrocytic processes by GFAP may be critical for neuron-glia interaction and CNS morphogenesis. This hypothesis is supported by findings that removal of GFAP in a human astrocytoma cell line by transfection with an antisense construct caused an impaired ability of the cells to extend glial processes upon neuronal induction. Such property is rescued by further transfection of GFAP-negative cells with a cDNA for GFAP (61).

Although other studies have postulated a requirement of GFAP for the formation of astrocytic processes, there is still some controversy about such mechanism. Pekny et al. (10) have recently reported that GFAP-deficient astrocytes in the primary cerebellar cultures could form normal processes in response to neurons. Thus GFAP might not be a sole prerequisite for the formation of astrocytic processes.

Another remarkable example of the relevance of GFAP expression in neuron-glia interaction regards regeneration of axonal growth after CNS lesions. The GFAP-glial scar formed after NS lesion is considered to be a barrier to regeneration of axon growth. Recently, by inhibiting GFAP translation with an antisense mRNA, Lefrançois et al. (62) demonstrated that GFAP expression is involved in the functional shift from neuritepromoting to neurite-inhibiting properties of reactive glia. Transfection of antisense GFAP mRNA not only blocked astroglial morphological changes but also made astrocytes permissive to neurite outgrowth. These results suggested that GFAP synthesis inhibition relieves the blockage of neurite outgrowth that normally is observed after a lesion. In contrast to this study, Wang et al. 
(63) did not find a correlation between the absence of GFAP and axonal outgrowth. They reported no increase in axon sprouting or long distance regeneration in the corticalspinal tract fibers of GFAP-/- mice.

Given the relevant role of GFAP during CNS development, as well as a factor in the reactive response to injury, understanding the mechanism of its modulation should be useful to elucidate some steps of NS physiology and pathology.

The close association between neurons and glia suggests that gene expression in these cell types is likely to be influenced by mutual interactions. Several lines of evidence indicate that glia influence the growth, migration and differentiation of neurons but the effect of neuronal cells on astrocytes is far from being well understood. Increasing evidence has been accumulated indicating that neurons are modulators of astrocyte gene expression and differentiation $(25,64,65)$.

Recently, we have demonstrated that neurons secrete brain region-specific soluble factors which induce GFAP gene promoter (25). We have used a transgenic mouse bearing part of the GFAP gene promoter linked to the $\beta$-galactosidase ( $\beta$-gal) reporter gene. Addition of cortical neurons or neuronal conditioned medium to transgenic astrocyte monolayers increased the number of $\beta$-gal astrocytes by $60 \%$, followed by an arrest of astrocytes from the cell cycle and induction of glial differentiation. Such event was dependent on the regional origin of the neurons in the brain since cerebral hemisphere neuronal factors were not able to induce the GFAP gene promoter of midbrain and cerebellum astrocytes. Indeed such results are in agreement with previous studies which have suggested that there are functional and regional differences between the various types of astrocytes possibly accounting for the complexity of neuron-glia interactions within the CNS $(66,67)$. Furthermore, GFAP is differently modulated by distinct brain regions. It is conceivable that differences in growth factor binding ability of the GFAP gene promoter from different regions could account for such diverse modulation.

Taken together, those results emphasize the complexity of neuron-glia interaction during CNS development and suggest that neurons may modulate the GFAP gene promoter and induce the astrocytic differentiation program. These data argue in favor of the possibility that modulation of intermediate filaments such as GFAP in astrocytes by growth factors might be implicated in cell differentiation as well as in cell-cell interactions during CNS development.

\section{Concluding remarks}

The organization of IF proteins into a complex network, their interaction with MTs and MFs to form the cytoskeleton and their appearance according to a highly specific developmental profile, all point to an important biological role of IF filaments. In this regard, the CNS has been extensively studied concerning IF functions. In this review, we highlighted the most recent works on IF gene regulation and its implications for astrocyte and CNS development.

The vertebrate nervous system is mainly formed by two distinct classes of cells which are neurons and glia. In this context, astrocytes comprise a heterogeneous family of morphologically and functionally distinct cells whose structural plasticity is mostly maintained by a filamentous network mainly consisting of vimentin and GFAP. Several lines of evidence indicate that the proliferation and differentiation of astrocytes are related in part to the modulation of GFAP expression during CNS development. Such expression is controlled by the regulation of gene activity, which is intrinsically dependent on several elements which compose its gene promoter.

Recently, by using transgenic animal models it has been possible to obtain more information about promoter activity and thus 
better understand the action of hormones and growth factors which modulate GFAP expression and its implications in astrocyte differentiation. The interactions between neuron and glial cells is essential to the harmonious development of the NS. Such interactions are based on junctional communication or on growth factors secreted by both cells which can mutually act on the metabolic pathways or more directly on gene activation, thus modulating cell fate.
Although we are still far from fully elucidating all questions involved in the physiology of normal and tumoral astrocytes, recent data regarding modulation of intermediate filaments such as GFAP by growth factors have greatly contributed to the understanding of astrocyte differentiation. Such works undoubtedly will help us to understand the more general mechanisms involved in CNS development and can open new perspectives for the control of neurologic diseases.

\section{References}

1. Galou M, Gao J, Humbert J M ericskay M, Li Z, Paulin D \& Vicart P (1997). The importance of intermediate filaments in the adaptation of tissues to mechanical stress: evidence from gene knockout studies. Biology of the Cell, 89: 85-97.

2. Georgatos SD, Gounari F, Goulielmos G \& Aebi U (1997). To bead or not to bead? Lens-specific intermediate filaments revisited. J oumal of Cell Science, 110 (Part 21): 2629-2634.

3. Yuan $Y$, Lee J A, Napier A \& Cole GJ (1997). Molecular cloning of a new intermediate filament protein expressed by radial glia and demonstration of alternative splicing in a novel heptad repeat region located in the carboxy-terminal tail domain. Molecular and Cellular Neurosciences, 10: 71-86.

4. Fuchs E \& Cleveland DW (1998). A structural scaffolding of intermediate filaments in health and disease. Science, 279: 514519.

5. Li Z, Mericskay M, Agbulut O, ButtlerBrowne G, Carlson L, Thornell L-E, Babinet C \& Paulin D (1997). Desmin is essential for the tensile strength and integrity of myofibrils but not for myogenic commitment, differentiation and fusion of skeletal muscle. J ournal of Cell Biology, 139: 1-16.

6. Zhu Q, Couillard-Despres S \& J ulien J P (1997). Delayed maturation of regenerating myelinated axons in mice lacking neurofilaments. Experimental Neurology, 148: 299-316.

7. Colucci-Guyon E, Portier M-M, Dunia I, Paulin D, Poumin S \& Babinet C (1994). Mice lacking vimentin develop and reproduce without an obvious phenotype. Cell, 79: 679-694.

8. Gomi H, Yokoyama T, Fujimoto K, Ikeda T,
Katoh A, Itoh T \& Itohara S (1995). Mice devoid of glial fibrillary acidic protein develop normally and are susceptible to scrapie prions. Neuron, 14: 29-41.

9. Liedtke W, Edelman W, Bieri PL, Chiu FC, Cowan NJ , Kucherlapati R \& Raine CS (1996). GFAP is necessary for the integrity of CNS white matter architecture and long-term maintenance of myelination. Neuron, 17: 607-615.

10. Pekny $M$, Eliasson $C$, Chien $C L$, Kindblom LG, Hamberger A \& Betsholtz C (1998). GFAP-deficient astrocytes are capable of stellation in vitro when cocultured with neurons and exhibit a reduced amount of intermediate filaments and an increased cell saturation density. Experimental Cell Research, 239: 332-343.

11. Barres BA (1991). New roles for glia. J ournal of Neuroscience, 11: 3685-3694.

12. Anton ES, Cameron RS \& Rakic P (1996). Role of neuron-glial junctional domain proteins in the maintenance and termination of neuronal migration across the embryonic cerebral wall. J ournal of Neuroscience, 16: 2283-2293.

13. Gomes FCA, Maia CG, Menezes J RL \& Moura Neto $\vee(1999)$. Cerebellar astrocytes treated by thyroid hormone induce neuronal proliferation. Glia, 25: 247-255.

14. Maxwell GD, Reid K, Elefanty A, Bartlett PF \& Murphy M (1996). Glial cell line derived neurotrophic factor promotes the development of adrenergic neurons in mouse neural crest cultures. Proceedings of the National Academy of Sciences, USA, 93: 13274-13279.

15. Bignami A \& Dahl D (1995). Gliosis. In: Kettenmann $\mathrm{H} \&$ Ransom BR (Editors), Neuroglia. Oxford University Press, New York, 843-858.

16. Temple S \& Qian X (1996). Vertebrate neural progenitor cells: subtypes and regulation. Current Opinion in Neurobiology, 6: 11-17.

17. Wofchuk ST \& Rodnight R (1995). Agedependent changes in the regulation by external calcium ions of the phosphorylation of glial fibrillary acidic protein in slices of rat hippocampus. Developmental Brain Research, 85: 181-186.

18. Pixley SK \& de Vellis J (1984). Transition between radial glia and mature astrocytes studied with a monoclonal antibody to vimentin. Developmental Brain Research, 15: 201-209.

19. Evans RM (1998). Vimentin: the conundrum of the intermediate filament gene family. BioEssays, 20: 79-86.

20. Moura-Neto V, Kryszke M-H, Li Z, Vicart P, Lilienbaum A \& Paulin D (1996). A 28bp negative element with multiple factorbinding activity controls expression of the vimentin-encoding gene. Gene, 168: 261266.

21. Galou M, Colucci-Guyon E, Ensergueix D, Ridet J L, Gimenez y Ribotta M, Privat A, Babinet C\& Dupouey P (1996). Disrupted glial fibrillary acidic protein network in astrocytes from vimentin knockout mice. J ournal of Cell Biology, 133: 853-863.

22. Eng LF, Vanderhaeghen J J , Bignami A \& Gerste B (1971). An acidic protein isolated from fibrous astrocytes. Brain Research, 28: 351-354.

23. Riol H, Fages C \& Tardy M (1992). Transcriptional regulation of glial fibrillary acidic protein (GFAP)-mRNA expression during postnatal development of mouse brain. J ournal of Neuroscience Research, 32: 79-85.

24. Laping NJ , Teter B, Nichols NR, Rozovsky I \& Finch CE (1994). Glial fibrillary acidic protein: regulation by hormones, cyto- 
kines, and growth factors. Brain Pathology, 1: 259-275.

25. Gomes FCA, Garcia-Abreu J, Galou M, Paulin D \& M oura Neto V (1999). Neurons induce glial fibrillary acidic protein (GFAP) gene promoter of astrocytes derived from transgenic mice. Glia (in press).

26. Liedtke W, Edelman W, Chiu FC, Kucherlapati R \& Raine CS (1998). Experimental autoimmune encephalomyelitis in mice lacking glial fibrillary acidic protein is characterized by a more severe clinical course and an infiltrative central nervous system lesion. American J oumal of $\mathrm{Pa}$ thology, 152: 251-259.

27. Gould EG, Frankfurt M, WestlindDanielsson A \& McEwen BS (1990). Developing forebrain astrocytes are sensitive to thyroid hormone. Glia, 3: 283-292.

28. Lima FRS, Trentin AG, Rosenthal D, Chagas C \& Moura Neto V (1997). Thyroid hormone induces protein secretion and morphological changes in astroglial cells with an increase in expression of glial fibrillary acidic protein. J ournal of Endocrinology, 154: 167-175.

29. Trentin AG, Gomes FCA, Lima FRS \& Moura Neto $\vee$ (1998). Thyroid hormone induces secretion of factors and progressive morphological changes on primary and subcultured astrocytes. In Vitro Cellular and Developmental Biology, 34: 280282.

30. Lima FRS, Gonçalves N, Gomes FCA, de Freitas MS \& Moura Neto V (1998). Thyroid hormone action on astroglial cells from distinct brain regions during development. International J ournal of Developmental Neuroscience, 16: 19-27.

31. Trentin AG \& Moura Neto V (1995). T3 affects cerebellar astrocyte proliferation, GFAP and fibronectin organization. NeuroReport, 6: 293-296.

32. O'Callaghan JP, Brinton RE \& McEwen BS (1989). Glucocorticoids regulate the concentration of glial fibrillary acidic protein throughout the brain. Brain Research, 494: 159-161.

33. De Nicola AF, Ferrini M, Gonzalez SL, Gonzalez Deniselle MC, Grillo CA, Piroli G, Saravia F \& de Kloet ET (1998). Regulation of gene expression by corticoid hormones in the brain and spinal cord. J ournal of Steroid and Biochemical Molecular Biology, 65: 253-272.

34. Melcangi RC, Magnaghi V, Cavarretta I, Riva MA \& Martini L (1997). Corticosteroid effects on gene expression of myelin basic protein in oligodendrocytes and of glial fibrillary acidic protein in type I astrocytes. J ournal of Neuroendocrinology, 9:
729-733.

35. Torres-Alerman I, Rejas MT, Pons S \& Garcia-Segura LM (1992). Estradiol promotes cell shape changes and glial fibrillary acidic protein redistribution in hypothalamic astrocytes in vitro: a neuronal mediated effect. Glia, 6: 180-187.

36. Day J R, Frank AT, O'Callaghan J P, J ones BC \& Anderson J E (1998). The effect of age and testosterone on the expression of glial fibrillary acidic protein in the rat cerebellum. Experimental Neurology, 151: 343-346.

37. Qian X, Davis AA, Goderie SK \& Temple S (1997). FGF2 concentration regulates the generation of neurons and glia from multipotent cortical stem cells. Neuron, 18: 81-93.

38. Kuhn HG, Winkler J , Kempermann G, Thal LJ \& Gage FH (1997). Epidermal growth factor and fibroblast growth factor-2 have different effects on neural progenitors in the adult rat brain. J ournal of Neuroscience, 17: 5820-5829.

39. Tao Y, Black IB \& DiCicco-Bloom E (1997). In vivo neurogenesis is inhibited by neutralizing antibodies to basic fibroblast growth factors. J ournal of Neurobiology, 33: 289-296.

40. CookJ L, Marcheselli V, Alam J, Deininger PL \& Bazan NG (1998). Temporal changes in gene expression following rat brain injury. Molecular Brain Research, 55: 9-19.

41. Gately S, Soff GA \& Brem S (1995). The potential role of basic fibroblast growth factor in the transformation of cultured primary human fetal astrocytes and the proliferation of human glioma (U-87) cells. Neurosurgery, 37: 1-10.

42. Holland EC \& Varmus HE (1998). Basic fibroblast growth factor induces cell migration and proliferation after glia-specific gene transfer in mice. Proceedings of the National Academy of Sciences, USA, 95: 1218-1223.

43. Kahn MA, Huang CJ , Caruso A, Barresi V, Nazarian R, Condorelli DF \& de Vellis J (1997). Ciliary neurotrophic factor activates J AK/Stat signal transduction cascade and induces transcriptional expression of glial fibrillary acidic protein in glial cells. J ournal of Neurochemistry, 68: 1413-1423.

44. Kahn MA, Ellison J A, Chang RP, Speight GJ \& de Vellis J (1997). Intracerebral injections of CNTF induce GFAP in S-100a brain cell population: the pattern of CNTFaR suggests an indirect mode of action. Developmental Brain Research, 98: 221233.

45. Clatterbuck RE, Prince DL \& Koliatsos VE
(1996). Ciliary neurotrophic factor stimulates the expression of glial fibrillary acidic protein by brain astrocytes in vivo. J ournal of Comparative Neurology, 369: 543-551.

46. Hudgins SN \& Levison SW (1998). Ciliary neurotrophic factor stimulates astroglial hypertrophy in vivo and in vitro. Experimental Neurology, 150: 171-182.

47. Balasingam $V$, Tejada-Berges $T$, Wright $E$, Bouckova R \& Yong WW (1994). Reactive astrogliosis in the neonatal mouse brain and its modulation by cytokines. J ournal of Neuroscience, 14: 846-856.

48. Oh YJ , Markelonis GJ \& Oh TH (1993). Effects of interleukin-1b and tumor necrosis factor- $\alpha$ on the expression of glial fibrillary acidic protein and transferrin in cultured astrocytes. Glia, 8: 77-86.

49. Campbell IL, Abraham CR, Masliah E, Kemper $\mathrm{P}$, Inglis J D, Oldstone MBA \& Mucke L (1993). Neurologic disease induced in transgenic mice by cerebral overexpression of interleukin 6. Proceedings of the National Academy of Sciences, USA, 90: 10061-10065.

50. Klein MA, Möller JC, J ones LL, Bluethmann H, Kreutzberg GW \& Raivich G (1997). Impaired neuroglial activation in interleukin-6 deficient mice. Glia, 19: 227233.

51. Koblar AS, Turnley AM, Classon BJ , Reid $\mathrm{KL}$, Ware CB, Cheema SS, Murphy M \& Barlett PF (1998). Neural precursor differentiation into astrocytes requires signaling through the leukemia inhibitory factor receptor. Proceedings of the National Academy of Sciences, USA, 95: 3178-3181.

52. Banner LR, Moayeri NN \& Patterson PH (1997). Leukemia inhibitory factor is expressed in astrocytes following cortical brain injury. Experimental Neurology, 147: 1-9.

53. Selmaj KW, Shafit-Zagardo B, Aquino DA, Farooq M, Raine CS, Norton WT \& Brosnan CF (1991). Tumor necrosis factor-induced proliferation of astrocytes from mature brain is associated with down regulation of glial fibrillary acidic protein mRNA. J ournal of Neurochemistry, 57: 823-830.

54. Murphy GM, Lee $Y L$, J ia $X-C, Y u A C H$, Majewska A, Song Y, Schmidt K \& Eng LF (1995). Tumor necrosis factor- $\alpha$ and basic fibroblast growth factor decrease glial fibrillary acidic protein and its encoding mRNA in astrocyte cultures and glioblastoma cells. J ournal of Neurochemistry, 65: 2716-2724.

55. Mehler MF, Marmur R, Gross R, Mabie PC, Zang Z, Papavasiliou A \& Kessler J A (1995). Cytokines regulate the cellular 
phenotype of developing neural lineage species. International J ournal of Developmental Neuroscience, 13: 213-240.

56. J ones LL, Kreutzberg GW \& Raivich G (1998). Transforming growth factor beta's 1, 2 and 3 inhibit proliferation of ramified microglia on an astrocyte monolayer. Brain Research, 795: 301-306.

57. Reilly J F, Maher PA \& Kumari VG (1998). Regulation of astrocyte GFAP expression by TGF-beta 1 and FGF-2. Glia, 22: 202210.

58. Shah NM, Groves AK \& Anderson DJ (1996). Alternative neural cresty cell fates are instructively promoted by TGFß superfamily members. Cell, 85: 331-343.

59. Gross RE, Mehler MF, Mabie PC, Zang Z, Santschi L \& Kessler J A (1996). Bone morphogenetic proteins promote astroglial lineage commitment by mammalian subventricular zone progenitor cells. Neuron, 17: 595-606.
60. Mabbie PC, Mehler MF, Marmur R Papavasiliou A, Song Q \& Kessler J A (1997). Bone morphogenetic proteins induce astroglial differentiation of oligodendroglial-astroglial progenitor cells. J ournal of Neuroscience, 17: 4112-4120.

61. Chen WJ \& Liem RK (1994). Reexpression of glial fibrillary acidic protein rescues the ability of astrocytoma cells to form processes in response to neurons. J ournal of Cell Biology, 127: 813-823.

62. Lefrançois $T$, Fages $C$, Peschanski $M$ \& Tardy M (1997). Neurite outgrowth associated with astroglial phenotypic changes induced by antisense glial fibrillary acidic protein (GFAP) mRNA in injured neuronastrocyte cocultures. J ournal of Neuroscience, 17: 4121-4128.

63. Wang X, Messing A \& David S (1997). Axonal and nonneuronal cell responses to spinal cord injury in mice lacking glial fibrillary acidic protein. Experimental Neurol- ogy, 148: 568-576.

64. Swanson RA, Liu J, Miller J W, Rothstein J D, Farrel K, Stein BA \& Longuemare MC (1997). Neuronal regulation of glutamate transporter subtype expression in astrocytes. J ournal of Neuroscience, 17: 932 940.

65. Hatten ME (1985). Neuronal regulation of astroglia morphology and proliferation in vitro. J ournal of Cell Biology, 100: 384396.

66. Garcia-Abreu J, Moura-Neto V, Carvalho SL \& Cavalcante LA (1995). Regionally specific properties of midbrain glia: I. Interactions with midbrain neurons. J ournal of Neuroscience Research, 40: 417-477.

67. Qian J , Bull MS \& Levitt P (1992). Targetderived astroglia regulate axonal outgrowth in a region-specific manner. Developmental Biology, 149: 278-294. 